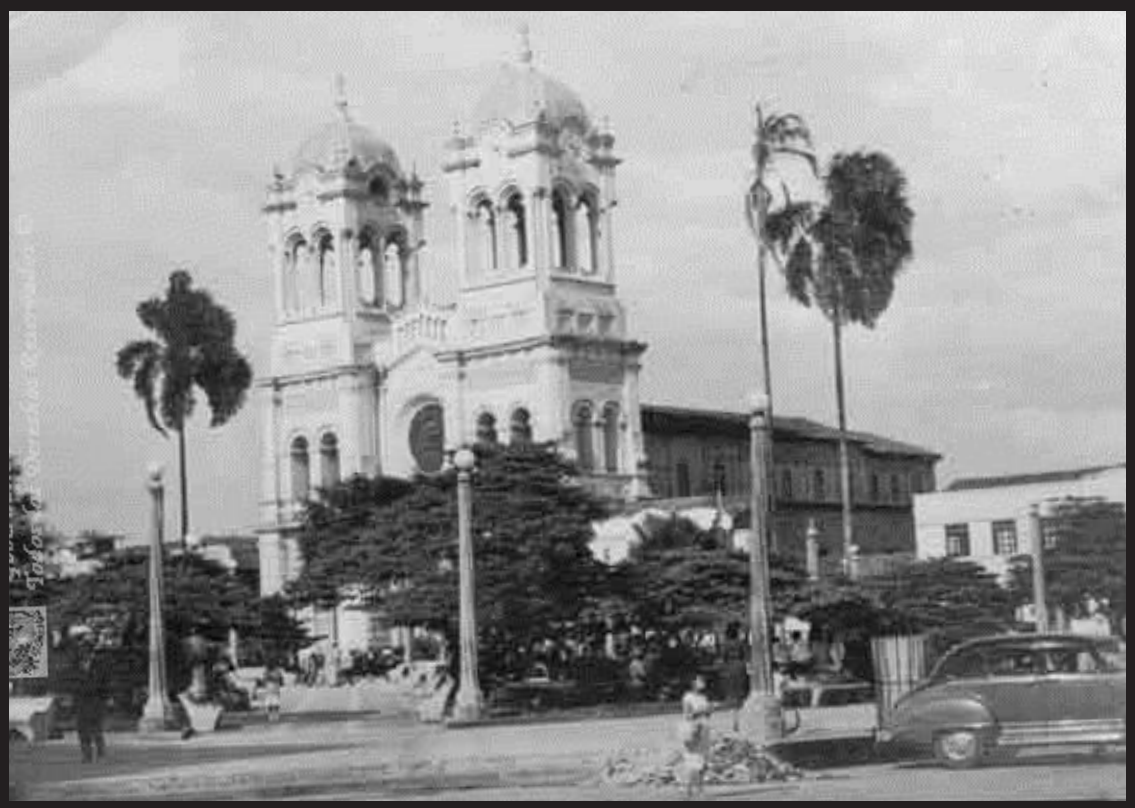

Panorámica de la iglesia y el parque (ca. 1940-1950) Fuente: Biblioteca Departamental Jorge Garcés Borrero, Archivo del Patrimonio Fotográfico y Fímico del Valle del Cauca (Santiago de Cali). 


\section{El camino a la especialización espacial: el asentamiento de la industria de las artes gráficas en el barrio San Nicolás. Santiago de Cali, 1940-1980 The path to space specialization: the settlement of graphic arts industry in the San Nicolás neighbourhood, Santiago de Cali, 1940-1980 O caminho da especialização espacial: o assentamento da indústria das artes gráficas no bairro San Nicolás. Santiago de Cali, 1940-1981}

Esteban Morera Aparicio

Katholische Universität Eichstätt-Ingolstadt

Eichstätt-Ingolstadt; Eberhard Karls

Universität Tübingen (Alemania)

estebanmorera@gmail.com
Este artículo fue elaborado en el marco de la investigación «Imprentas y espacio urbano en Cali. Caracterización histórica del barrio San Nicolás desde la industria de la imprenta entre las décadas de 1920 y 1980 », del Departamento de Humanidades de la Universidad ICESI (Santiago de Cali).

\section{Resumen}

El barrio San Nicolás, reconocido por agrupar la mayor parte de la industria de las artes gráficas, fue uno de los casos más representativos de los diferentes fenómenos que caracterizaron a la ciudad de Cali durante el siglo XX. A comienzos de tal siglo, San Nicolás arrastraba una herencia decimonónica como barrio de artesanos que lo llevó a convertirse, con los años, en una de las zonas con mayor presencia industrial y comercial dentro de la ciudad. Ante el crecimiento acelerado, el barrio pasó de ser periferia a centro de la ciudad, y en las décadas de 1960 y 1970 llegó a concentrar la mayor parte de la industria editorial caleña. En este sentido, se conjugaron los elementos que posibilitaron, por un lado, el establecimiento temprano de imprentas grandes en la zona de influencia del barrio y, por otro, la capacidad de emprendimiento y organizativa de los pequeños y medianos impresores, para aprovechar los espacios que abrió la gran industria.

\section{Palabras clave}

Santiago de Cali; barrio San Nicolás; imprentas; industria de las artes gráficas; desarrollo urbano; siglo XX

\section{Abstract}

The neighbourhood of San Nicolás in Cali, Colombia, has been the centre of the graphic arts industry of the city and, thus, is an ideal place to observe the industrial and cultural development of Cali in the $20^{\text {th }}$ century. Starting as a zone with a significant craft heritage in the early $20^{\text {th }}$ century, San Nicolás, where most of the city's editorial industry has been concentrated, more recently evolved into an area of major industrial and commercial activity; it was transformed by Cali's accelerated growth in the 1960s and 1970s and is not longer considered peripheral in terms of the urban area. Such factors, together with the organizational abilities of small and medium printing entrepreneurs, have contributed to the establishment in the neighbourhood of printing companies of scale that have taken advantage of the opportunities created by the editorial business.

\section{Keywords}

Santiago de Cali; San Nicolás neighbourhood; printing industry; the graphic arts industry; urban development; 20th century

\section{Resumo}

O bairro San Nicolás, reconhecido por agrupar a maior parte da indústria das artes gráficas, foi um dos casos mais representativos dos diferentes fenômenos que caracterizaram Cali durante o século XX. No início deste século, San Nicolás arrastava uma herança oitocentista como bairro de artesãos que levou-o para se converter, ao longo dos anos, em uma das áreas com maior presença industrial e comercial dentro da cidade. Ante o crescimento acelerado, o bairro virou de periferia para centro da cidade, e nas décadas de 1960 e 1970 conseguiu concentrar a maior parte da indústria editorial caleña. Neste sentido, conjugaram-se os elementos que possibilitaram, por um lado, o estabelecimento temporão de empresas grandes de impressão na área de influência do bairro e, por outro, a capacidade de empreendedorismo e organizativa das impressoras pequenas e médias, para tirar proveito dos espaços que a indústria abriu.

\section{Palavras-chave}

Santiago de Cali; bairro San Nicolás; impressoras; indústria das artes gráficas; desenvolvimento urbano; século XX

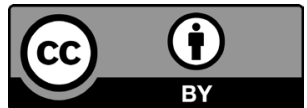




\section{Introducción}

En la década de 1940, Santiago de Cali había roto todos los récords de crecimiento urbano y poblacional de su historia. Durante las primeras décadas del siglo XX, la ciudad ya había duplicado el tamaño que tuvo durante su larga vida colonial y su primer siglo de vida republicana. Sin embargo, este crecimiento podría ser considerado menor, con relación a la evolución que se presentó durante las décadas siguientes.

Las consecuencias de este crecimiento desbordante van a repercutir en la organización que la ciudad había configurado a lo largo de su historia, marcada por la estructuración colonial. Una pequeña ciudad que se desarrolló en torno de la plaza central, cuya cercanía a este punto de referencia representaba, dentro de la dinámica de la época, mayor o menor prestigio social, así como mayor o menor vinculación a la vida económica. El trasegar de la pequeña ciudad consolidó una organización urbana que congregó las principales actividades políticas, económicas y culturales en el centro, con apenas incipientes iniciativas de agrupación por parte de ciertos sectores económicos. Sin embargo, la entrada en el siglo XX representó una transformación radical de la estructura económica de Cali, que reconfiguró su organización urbana.

En este contexto, las agrupaciones urbanas jugaron un papel fundamental en la medida en que crecieron la economía y la población, y la ciudad necesitó de una organización urbana especializada por sectores. En este aspecto no siempre resultó fundamental la intención de las políticas públicas de redistribuir las actividades económicas por sectores, sino que, en muchos casos, fue la dinámica económica de la ciudad y de los diferentes sectores económicos lo que determinó la suerte urbana de los diferentes barrios de la ciudad.

El barrio San Nicolás, a lo largo de su historia, tuvo un papel preponderante dentro de la dinámica económica caleña. A partir de las décadas de 1970 y 1980 empezó a reconocerse a este barrio como el espacio de concentración de las imprentas y de la actividad editorial, por excelencia, de la ciudad de Cali. Sin embargo, vale la pena preguntarse si desde sus inicios San Nicolás fue un barrio de impresores, y plantearse, así mismo, en qué momento empezó a tener esta particularidad y por qué motivo. A continuación, hablaré sobre el desarrollo del barrio durante las décadas de 1940 y 1970, al tiempo que abordaré la evolución de la industria editorial y el proceso mediante el cual la mayor parte de esta se fue asentando en el barrio.

\section{Santiago de Cali en el siglo $\mathrm{XX}$, una ciudad en estado de transición}

Una de las características más importantes de la ciudad, como objeto de análisis histórico, es que ha sido una constante en la historia de la humanidad. Su importancia radica en que es el reflejo de la forma como los seres humanos han transformado el espacio físico para su propio beneficio, convirtiéndolo de esta manera, en un espacio histórico ${ }^{1}$. En el caso de las ciudades latinoamericanas, los flujos migratorios transformaron las viejas estructuras coloniales bajo las cuales fueron fundadas. En muchos casos, este proceso, que se ha catalogado «de tránsito» entre la ciudad colonial y la ciudad burguesa o moderna, comenzó a desarrollarse en el siglo XIX, pero en el caso de Cali, el crecimiento demográfico empezó a presentarse de manera exponencial a partir de las primeras décadas del siglo XX. Como veremos, no se vivió una ruptura radical entre un escenario y otro. Muchos aspectos de la organización urbana, de la infraestructura, de la cultura

1 Germán Mejía Pavony, Los años del cambio. Historia urbana de Bogotá. 1820-1910 (Bogotá: Pontificia Universidad Javeriana, 2000), 15-25. Sobre el tema de la ciudad como objeto de estudio histórico se ha escrito mucho, desde los clásicos de Braudel y Febvre hasta los estudios de los profesores Aprile-Gniset y Fabio Zambrano en Colombia: Jacques Aprile-Gniset, La ciudad colombiana: Siglo XIX y Siglo XX (Bogotá: Banco Popular, 1992). Uno de los trabajos más interesantes es el del profesor Mejía Pavony, quien aborda el proceso de transformación que sufrió la ciudad de Bogotá en el siglo XIX, presentando lo que él considera el tránsito entre la ciudad colonial y la ciudad moderna. Mejía también analiza tres características fundamentales que centran la discusión sobre la ciudad dentro de la disciplina histórica: su continuidad en la historia, su capacidad ordenadora del territorio, y su capacidad de congregación de los seres humanos y de interacción de los poderes. En este sentido, la ciudad no es un simple lugar de contención de los fenómenos humanos, sino también un resultado del nudo de relaciones sociales que, al espacializarse, dan paso a un espacio humanamente construido. 
y de la estructura social, arrastrados desde el siglo XIX, van a lograr sobrevivir durante el periodo en el que se concentra la investigación (19401980). Sin embargo, van a presentarse procesos de transformación muy fuertes en todas las esferas de la sociedad.

Veremos entonces cómo sobre una estructura colonial de calles pequeñas, retícula y centralización, empezaron a desarrollarse nuevos elementos de una sociedad burguesa. Fue así como un sistema fuertemente estamental ${ }^{2}$ se encontró frente al avance de sectores industriales y comerciales que pugnaban por imponerse 3 . En medio de todo ello, ese crecimiento desbordante constituyó un reto para la ciudad que, entre sus estrechas estructuras urbanas coloniales, tuvo que albergar las consecuencias de estos procesos de transformación.

Es así como, al empezar la década de 1940, la ciudad de Cali y el todavía joven Valle del Cauca ${ }^{4}$ habían logrado consolidar el proyecto político que concibió el nacimiento del departamento y la designación de Cali como capital de la nueva unidad político-administrativa. Este proyecto, que articuló en términos políticos, administrativos y de infraestructura el Puerto de Buenaventura, las zonas industriales del departamento y las economías agroindustriales, se sumó a la serie

2 En este punto siempre vale la pena recordar el trabajo de Jacques Aprile-Gniset, cuando en su libro La ciudad colombiana dice que para el siglo XIX «La casta urbana dominante [caleña] había elaborado cien años atrás su propio sistema de valores y estatus social. Mantenía intacto un código de prestigio y honorabilidad falsificando la realidad y adulterando calificativos: sus integrantes se adjudicaban títulos de "capitanes" cuando eran ganaderos, se afirmaban españoles pero no eran ni siquiera criollos, se pretendían nobles cuando eran plebeyos, y fingían ser blancos siendo todos "mestizos", situación que iba a variar con la modificación de esquema político y económico del país, el final de un sistema económico basado en el esclavismo y la llegada de nuevas élites industriales y comerciales al poder» Aprile-Gniset, La ciudad colombiana, 653-654.

3 Eduardo Sáenz Rovner, La ofensiva empresarial: industriales, políticos y violencia en los años 40 en Colombia (Bogotá: Tercer Mundo, 1992).

4 La fundación del departamento de Valle del Cauca y el establecimiento de Santiago de Cali como su capital se dio el 16 de abril de 1910, como culminación de un proceso político que adelantaron las élites de la región vallecaucana y que desligó el territorio de la nueva unidad político-administrativa del antiguo Estado Soberano del Cauca. Para el análisis de este proceso son fundamentales los trabajos de la profesora Aura Hurtado. Véase, por ejemplo: Aura Hurtado, «Opinión pública y formación del departamento del Valle, 1903-1910», CS 7 (2011): 161-192. de fenómenos que transformaron, en pocos años, a la ciudad de Cali; un lugar que hasta la entrada del siglo XX había sido una pequeña población con no más de veinte mil habitantes. Para la década de 1940, la ciudad ya había duplicado su población (véase Tabla 1).

Con relación a la población, estos primeros cuarenta años del siglo XX fueron claves para convertir a Cali en una de las ciudades más importantes del país; de hecho, se consolidó como la tercera más poblada. A mediados del siglo XX, Cali contaba con una población de alrededor de trescientos mil habitantes; diez veces más que al inicio del siglo. Al finalizar la centuria, Cali ya contaba con dos millones de habitantes, es decir que, en menos de 100 años, pasó de ser una ciudad de treinta mil habitantes a una de dos millones.

Tabla 1.

Población de Cali siglo XX

\begin{tabular}{|l|l|l|}
\hline Año & Habitantes & $\begin{array}{l}\text { Participación } \\
\text { de Cali en el Valle del } \\
\text { Cauca }\end{array}$ \\
\hline 1905 & 31.712 & $12 \%$ \\
\hline 1912 & 37.610 & $13,5 \%$ \\
\hline 1918 & 57.480 & $16,8 \%$ \\
\hline 1928 & 83.791 & $17 \%$ \\
\hline 1938 & 128.638 & $17,5 \%$ \\
\hline 1951 & 328.841 & $25,7 \%$ \\
\hline 1964 & 713.878 & $36,8 \%$ \\
\hline 1973 & 991.549 & $41,4 \%$ \\
\hline 1985 & 1.429 .026 & $47,2 \%$ \\
\hline
\end{tabular}

Fuente: Fernando Urrea Giraldo, «Transformaciones sociodemográficas y grupos socioraciales en Cali, siglo XX e inicios del siglo XXI», en Historia de Cali siglo XX, ed. Gilberto Loaiza Cano (Cali: Universidad del Valle, 2012), 151.

Igualmente, fue notable el incremento de la población a nivel regional. Los cascos urbanos de las ciudades de la región también aumentaron notablemente su población durante este periodo ${ }^{5}$, un aspecto impulsado no solo por el auge comercial e industrial, sino también por el crecimiento

\footnotetext{
5 Sin embargo, y como lo podemos observar en Tabla 1, este aumento de las ciudades del entorno regional de Cali no se comparaba con los niveles que estaba experimentando la capital vallecaucana. Esto es palpable al ver la participación de la población caleña en el Valle del Cauca, que al iniciar el siglo representa el $12 \%$, y ya en la década de 1980 va a ser casi del $50 \%$. Es decir que, para la década de 1980, la mitad de la población vallecaucana se encontraba en Cali.
} 
desbordante de la gran propiedad — sobre la pequeña y la mediana - que se presentó en las zonas agrícolas, y que en muchos casos fue impulsado violentamente ${ }^{6}$.

El fenómeno demográfico que experimentó la ciudad de Cali en el siglo XX condicionó su proceso de formación urbana y económica, teniendo en cuenta la manera acelerada en que este se presentó, con relación a como había sido históricamente el crecimiento poblacional. Santiago de Cali fue fundada alrededor del año 1536, y durante el periodo colonial y los primeros años de vida republicana contó con una dinámica social generada, en gran parte, por la economía de las haciendas - de fuertes concentraciones de tierra- y un flujo comercial determinado por la economía minera. En la medida que se fue formando la nación colombiana y que la república fue definiendo su vocación económica, la ciudad de Cali fue cobrando mayor relevancia dentro del esquema productivo nacional.
Como era de esperarse, los cambios económicos y demográficos incidieron en el desarrollo urbano de la ciudad. Ya para la década de 1940 - las siguientes referencias se pueden ver en la Figura 1-, la histórica concentración que había caracterizado a Cali, se fue rompiendo con el nacimiento de ciertos lugares que se encontraban desconectados del casco urbano ${ }^{7}$, como los talleres del Ferrocarril en Chipichape, al norte de la ciudad, que además era el camino al eje industrial que se consolidaba entre Cali y Yumbo; al occidente estaba la vía que conducía al mar, uno de los principales focos para el desarrollo de Cali; hacia el sur se encontraba el barrio San Fernando y en él las instalaciones deportivas, el estadio y el hipódromo - en medio de estos dos se había situado el Templete Eucarístico-; y al oriente el campo de aviación El Guabito, sector en el que en la actualidad se halla la base aérea (carrera 8 con calle 44), que también era el camino a Juanchito y una de las principales vías de abastecimiento de la ciudad por el comercio

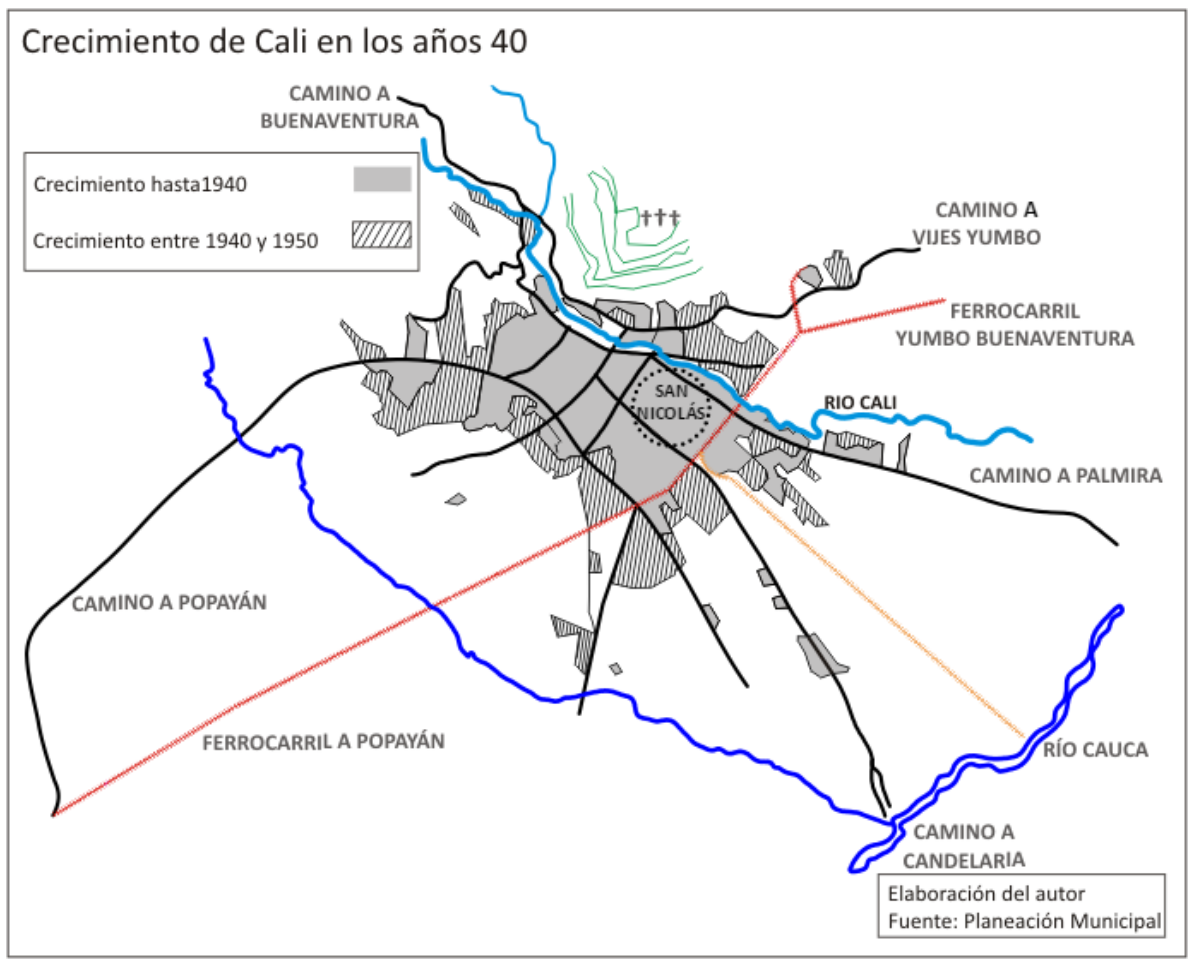

Figura 1.

Crecimiento de Cali en los años 40
6 Salomón Kalmanovitz, «El desarrollo histórico del campo colombiano», en Colombia Hoy, ed. Jorge Orlando Melo González (Santa Fe de Bogotá: Presidencia de la República, 1996).
7 Planeación Municipal, Proceso histórico, periodos de formación de la ciudad (Cali: Alcaldía de Santiago de Cali, 1977). 
desarrollado en torno al río Cauca. El camino a Juanchito entraba a la ciudad pasando por E1 Vallano, posteriormente conocido como San Nicolás, barrio tradicionalmente habitado por artesanos, pulperos y peones ${ }^{8}$.

Este proceso derivó en una crisis en la infraestructura de la ciudad. Los pobladores de la época eran conscientes del crecimiento urbano que se estaba viviendo. Por su parte, los periódicos locales, al tiempo que informaban con agrado sobre el movimiento inmobiliario de la ciudad, planteaban su preocupación por la desorganización con que se daba ${ }^{9}$. Todos los días se reseñaban la gran cantidad de problemas que se estaban presentando en Cali, una ciudad que no estaba lista para el crecimiento: sin andenes amplios, sin calles señalizadas ni propicias para el trasporte automotor $\mathrm{y}$, además, con una densidad poblacional que cada vez era más alta; y con gentes que se encontraban poco preparadas para la nueva dinámica urbana ${ }^{10}$.

Dentro de este fenómeno, el barrio San Nicolás tuvo importancia histórica, porque además de ser la entrada al casco urbano de quienes provenían de Palmira y Juanchito - $\mathrm{y}$, por ende, del aeropuerto-,

8 Como lo señala el profesor Ramiro Bonilla, un análisis de los modelos de trazado de la ciudad para el siglo XX muestra que existían dos principalmente: el primero, derivado de las disposiciones fundacionales de las Leyes de Indias, es el que se desarrolla bajo el concepto de cuadrícula. El segundo parte de la adaptación a las diferentes características topográficas de los espacios donde se construyen los barrios -lugares situados en las montañas o cercanos a los ríos-. Dentro del primer modelo se encuentran los barrios de la parte plana de la ciudad, entre ellos San Nicolás, donde se podrá observar la estructura de la plaza central y la parroquia. Estas disposiciones, marcadas por la lógica de la vida colonial que se arrastraban incluso hasta entrado el siglo $X X$, van a dejar una huella tangible en la organización de los barrios más antiguos de la ciudad. No obstante, para las décadas de 1930 y 1940, el concepto de «parroquia» va a ser desplazado por el de «barrio», como reflejo del triunfo de una idea más laica de los procesos de urbanización en la ciudad. Véase Ramiro Bonilla, «Modelos urbanísticos de Cali en el siglo XX», en Historia de Cali, siglo XX, ed. Gilberto Loaiza (Santiago de Cali: Universidad del Valle, 2012), 25-85.

9 Véase Esteban Morera Aparicio, «Vida pública en Cali y el impacto del gaitanismo en la ciudad (1945-1950)» (Tesis de pregrado, Universidad del Valle, 2011), 35-53.

10 Es importante resaltar que fue precisamente en 1945 que se comenzaron a establecer los primeros espacios de colonización urbana popular masiva, con el nacimiento de Terrón Colorado, Junín y Siloé, que se ubicaba en la mina artesanal de carbón y al cual llegaron desplazados del antiguo Caldas. Esto se articuló, además, con diferentes acciones políticas, encabezadas por los abanderados de la lucha por la vivienda popular urbana, es decir, el concejal comunista Julio Rincón y el liberal Alfonso Barberena. Véase: Jacques Aprile-Gniset, La ciudad colombiana, 706. era un espacio de condensación de todas estas transformaciones. San Nicolás, como barrio popular, se consolidó como un «espacio mediador» ${ }^{11}$ entre las culturas campesinas y las de los habitantes ya establecidos; este conservó elementos de la Cali decimonónica, pero al mismo tiempo adoptó elementos de la ciudad de grandes proporciones en la que se estaba transformando. En una dimensión más amplia, veremos en San Nicolás el encuentro de las estructuras artesanales con las pequeñas, medianas y grandes industrias, así como la llegada de las multinacionales a la ciudad.

\section{Consideraciones económicas del periodo 1940-1970}

La victoria política lograda por la clase dirigente de Cali frente a otros sectores del poder regional - con la creación de departamento del Valle del Caucaincidió positivamente en el crecimiento e importancia de la ciudad como centro administrativo del sector público regional. Cabe anotar, no obstante, que el factor determinante fue la forma como se transformó en el punto nodal de la conexión entre la región andina y el Pacífico. Rápidamente el puerto de Buenaventura se consolidó como el principal puerto para la exportación del café, y Cali se convirtió en paso obligado de las mercancías que se trasladaban por esa ruta. El comercio de equipos e insumos para el desarrollo de las economías cafetera y azucarera, así como las exportaciones, fueron creando un importante movimiento económico - logística, transporte, comercio, acopio, almacenamiento, finanzas, hotelería, entre otras actividades-. Una vez el país comenzó a transformar su estructura — de una economía agroexportadora a una fase de industrialización-, Cali se convirtió en el foco económico más importante del suroccidente colombiano, gracias a su condición de punto nodal del Pacífico.

Al iniciar el siglo, la ciudad era fuerte en producción primaria — agricultura, caza, pesca, sevicultura-, lo que fue cediendo espacio frente a los sectores comercial y financiero, de transporte y de servicios.

\footnotetext{
11 Véase Alejandro Ulloa, «Modernidad, barrio popular y músicas mulatas: el samba brasilero y el tango ríoplatense», Nexus, Comunicación, n. 17 (2015): 230.
} 
Esta evolución muestra cómo Cali transformó su vocación agrícola-campesina en la medida que se adentraba en el siglo XX. Mientras que, en las décadas de 1920, casi un tercio de la población empleada se encontraba dentro del sector primario, 30 años después, en la década de 1950, tan solo el $4 \%$ de la población empleada activa se desempeñaba en esta actividad económica, y en la década de 1970 únicamente el 2\% (véase Tabla 2).

Entre las décadas de 1940 y 1970, al tiempo que el sector primario presentó un decrecimiento, tanto el sector comercial y financiero como el de servicios experimentaron crecimientos formidables, al punto de convertirse en las principales actividades económicas de la ciudad, junto a la industria manufacturera, que tuvo un crecimiento proporcional al poblacional. Durante el periodo de estudio, un tercio de la población caleña se encontraba empleada por la industria manufacturera (véase Tabla 2). la ciudad. Estas transformaciones evidencian una constante pugna entre la cultura campesina y la citadina, que en un escenario más amplio y con una gran variedad de matices, se presentó como la tensión entre la tradición y la modernidad ${ }^{12}$.

El análisis de la evolución industrial entre las décadas de 1940 y 1970 arroja información muy interesante con relación al sector manufacturero ${ }^{13}$. $\mathrm{Al}$ inicio de la década de 1940 dominaban las industrias de alimentos, con una participación en la empleabilidad del 12,5\%), de textiles (con el 20\%), y de productos de metal (con el 10\%). Se puede notar un predominio de la participación de la industria textil dentro del grueso de la industria manufacturera. Sin embargo, este sector concentraba su fortaleza en menor cantidad de establecimientos, es decir, en el mundo textil prevalecía la gran industria sobre la pequeña y la mediana. Lo contrario ocurría en la industria alimenticia, que contaba con 10 veces más establecimientos que la

Tabla 2.

Porcentaje de Empleo por actividades económicas

\begin{tabular}{|c|c|c|c|c|c|}
\hline Rama de actividad & 1918 & 1938 & 1951 & 1964 & 1973 \\
\hline Producción primaria & 31,2 & 20,6 & 4,4 & 2,7 & 1,9 \\
\hline a. Agricultura, caza, pesca, sevicultura & & & 3,3 & 2,1 & 1,5 \\
\hline b. Minas y canteras & & & 1,1 & 0,6 & 0,4 \\
\hline Industria manufacturera & 28,3 & 27,8 & 31 & 24,9 & 28,6 \\
\hline Construcción & & 8,1 & 11,1 & 9,5 & 8,3 \\
\hline Comercio y finanzas & 13 & 14,1 & 16,9 & 21,3 & 24,8 \\
\hline a. Comercio, hoteles y restaurantes & & & 14 & 18,3 & 21,7 \\
\hline b. Establecimientos financieros & & & 2,9 & 3 & 3,1 \\
\hline Transporte y comunicaciones & 1,9 & 5,4 & 9 & 7,1 & 5,9 \\
\hline Servicios diversos & 13,5 & 21,2 & 27,6 & 29,5 & 30,5 \\
\hline a. Electricidad, gas y agua & & & 0,6 & 0,8 & 1 \\
\hline b. Servicios personales & & & 27 & 28,7 & 29,5 \\
\hline Otras actividades & 12,1 & 2,8 & & 5 & \\
\hline
\end{tabular}

Todo esto arroja transformaciones drásticas, no solo en las estructuras económicas, sino una reconfiguración del campo social. El cambio de una sociedad ahora dedicada a actividades comerciales e industriales, contrastaba con su reciente pasado campesino-agricultor y con las fuertes oleadas migratorias que desde las zonas rurales estaban impactando
12 Véase Oscar Almario García, «Cali y el Valle del Cauca: configuración moderna y reconfiguración contemporánea de la región y la ciudad región», en Historia de Cali siglo XX, tomo II, ed. Gilberto Loaiza (Cali: Universidad del Valle, 2012), 70-93.

13 Para abordar este punto, analicé los censos industriales que se realizaron en los años 1944, 1954 y 1971. Véanse: DANE, Censo Industrial 1944 (Bogotá: Departamento Administrativo Nacional de Estadística, 1945); DANE, Censo Industrial 1954 (Bogotá: Departamento Administrativo Nacional de Estadística, 1955); DANE, Censo industrial 1971. 
industria textil, pero generaba la mitad de puestos de trabajo. Llama la atención el caso de la industria del cuero que, junto a los textiles, fue una de las industrias con mayor cantidad de establecimientos, pero cuya participación en la empleabilidad resultó inferior a la de otros sectores. En el sector de la industria del cuero proliferaron los locales con empresas familiares que contaban con un promedio de 10 empleados por local; en los sectores del vestuario y de la madera pasaba algo similar: pues predominaban las pequeñas y medianas empresas (véase Tabla 3).

La evolución de la industria en las siguientes décadas mostró cómo la predominancia de la industria textil fue decayendo de manera drástica, para darle paso a la industria del vestuario. Si bien aumentó la generación de empleo por parte del sector textil - en cantidad de personas

Tabla 3.

Evolución de la industria en el eje Cali - Yumbo

\begin{tabular}{|c|c|c|c|c|c|c|c|c|c|}
\hline & & 1945 & & & 1965 & & & 1974 & \\
\hline & $\begin{array}{l}\text { Estableci- } \\
\text { mientos }\end{array}$ & $\begin{array}{l}\text { Emplea- } \\
\text { bilidad }\end{array}$ & $\begin{array}{l}\text { Empleabi- } \\
\text { lidad \% }\end{array}$ & Estab. & Emplea. & Emplea. \% & Estab. & Emplea. & Emplea. \% \\
\hline Alimentos & 62 & 1.258 & 12,53 & 154 & 3.209 & 8,64 & 87 & 5.843 & 10,54 \\
\hline Bebidas & 16 & 541 & 5,39 & 27 & 1.298 & 3,49 & 10 & 1.266 & 2,28 \\
\hline Tabaco & 4 & 287 & 2,86 & 3 & 311 & 0,84 & 2 & 329 & 0,59 \\
\hline Textiles & 5 & 1.997 & 19,89 & 38 & 3.561 & 9,58 & 27 & 6.043 & 10,9 \\
\hline Vestuario & 55 & 898 & 8,94 & 242 & 4.661 & 12,54 & 108 & 5.575 & 10,05 \\
\hline Madera & 59 & 557 & 5,55 & 77 & 1.262 & 3,4 & 28 & 964 & 1,74 \\
\hline $\begin{array}{l}\text { Imprentas y } \\
\text { editoriales }\end{array}$ & 21 & 576 & 5,74 & 59 & 2.573 & 6,92 & 36 & 4.246 & 7,66 \\
\hline $\begin{array}{l}\text { Metales } \\
\text { preciosos }\end{array}$ & 9 & 163 & 1,62 & & & & & & \\
\hline Papel & 9 & 197 & 1,96 & 27 & 2.818 & 7,58 & 42 & 4.426 & 7,98 \\
\hline Cuero & 62 & 753 & 7,5 & 17 & 481 & 1,29 & 10 & 946 & 1,71 \\
\hline $\begin{array}{l}\text { Productos de } \\
\text { caucho }\end{array}$ & 5 & 345 & 3,44 & 9 & 2.525 & 6,8 & 8 & 2.472 & 4,46 \\
\hline $\begin{array}{l}\text { Productos } \\
\text { químicos }\end{array}$ & 17 & 448 & 4,46 & 67 & 4.687 & 12,61 & 52 & 7.452 & 13,44 \\
\hline $\begin{array}{l}\text { Minerales no } \\
\text { metálicos }\end{array}$ & 16 & 859 & 8,5 & 74 & 2.274 & 6,12 & 26 & 2.873 & 5,18 \\
\hline $\begin{array}{l}\text { Industria } \\
\text { metálica } \\
\text { básica }\end{array}$ & & & & 4 & 344 & 0,93 & 10 & 1.470 & 2,65 \\
\hline $\begin{array}{l}\text { Productos de } \\
\text { metal }\end{array}$ & 40 & 1.054 & 10,5 & 102 & 2.214 & 5,96 & 72 & 3.646 & 6,58 \\
\hline $\begin{array}{l}\text { Maquinaria } \\
\text { no eléctrica }\end{array}$ & & & & 39 & 493 & 1,33 & 35 & 1.503 & 2,71 \\
\hline $\begin{array}{l}\text { Maquinaria } \\
\text { y artefactos } \\
\text { electrónicos }\end{array}$ & & & & 38 & 1.277 & 3,44 & 21 & 2.219 & 4 \\
\hline $\begin{array}{l}\text { Material de } \\
\text { transporte }\end{array}$ & & & & 67 & 2.082 & 5,6 & 14 & 1.762 & 3,18 \\
\hline Otros & 6 & 112 & 1,12 & 49 & 1.088 & 2,93 & 49 & 2.416 & 4,36 \\
\hline Total & & 10.045 & 100 & 1.093 & 37.158 & 100 & 637 & 55.451 & 100 \\
\hline
\end{tabular}

El camino a la especialización espacial: el asentamiento de la industria de las artes gráficas / Esteban Morera Aparicio / 99 
empleadas-, su participación en la tasa de empleo disminuyó a la mitad. Esto como resultado de la expansión económica de otros sectores, del crecimiento poblacional exponencial y de un cambio de dinámica del sector, ya que en la medida que contó con mayor cantidad de establecimientos, su participación en la empleabilidad disminuyó. Algo muy similar a lo que se presentó en la industria metalúrgica, que aumentó la cantidad de personal ocupado, así como la cantidad de establecimientos, pero con una disminución en la participación de la empleabilidad. Dos casos llamativos fueron los de la industria química y la del papel, ya que ambas presentaron un crecimiento alto en el periodo de análisis. En estos casos fueron claves dos aspectos, por un lado, el aprovechamiento del entorno económico ante la articulación con la industria de la caña, y las ventajas comparativas de la cercanía con el puerto de Buenaventura - teniendo en cuenta la importancia que jugaba la importación de insumos dentro de estos dos sectores-. Este último aspecto también fue aprovechado por otros sectores; tal fue el caso de la industria de la maquinaria que, con una participación significativa en la década de 1960, se consolidó en la de 1970.

Toda esta dinámica económica contó con un desarrollo marcado por la estabilidad de la industria de los alimentos, una estabilidad que la mayoría de las veces se impuso al crecimiento. De la misma manera, otros dos sectores también se caracterizaron por una fuerte estabilidad. Se trata de dos sectores que nos interesan por el papel que jugaron en el barrio San Nicolás: la industria del caucho y de las imprentas. A la industria del caucho nos acercaremos más adelante, por ahora, nos centraremos en la industria editorial, eje central del presente trabajo.

\section{La industria de las artes gráficas}

Durante el periodo comprendido entre las décadas de 1940 y 1970, la industria de las artes gráficas presentó, como ya se ha mencionado, un crecimiento constante pero no acelerado. Dicho periodo se caracterizó por ser una etapa bastante estable para la industria editorial dentro del sector manufacturero. Esto no quiere decir que la industria no haya crecido, lo que se observa es que su expansión fue paralela al crecimiento general de la economía. De hecho, la industria editorial caleña era una de las más fuertes del país: ocupaba el segundo lugar, superada tan solo por la industria editorial bogotan $a^{14}$.

Desde inicios de la década de 1940, la industria editorial caleña estuvo marcada por la proliferación de pequeños y medianos establecimientos. También se destacó la presencia de una gran empresa que iba jalonando el crecimiento de la industria en la ciudad, Carvajal S.A., una de las compañías más importantes de la región ${ }^{15}$.

Durante el periodo de estudio, este subsector industrial ocupó, en promedio, al 7\% de las personas empleadas dentro del sector manufacturero —a mediados de la década de 1940 al 5,74\% ${ }^{16}$ y en la década de 1970 al 7,66\%17—. En la década de 1960, la industria editorial aumentó en número de establecimientos y de empleados. En contraste, durante la década de 1970, el subsector duplicó el número de empleados y disminuyó casi en la mitad el número de establecimientos, lo que muestra que el desarrollo de las artes gráficas durante el periodo de estudio concentró a un mayor número de empleados en un menor número de establecimientos, lo que permite ver una mayor fortaleza de la mediana y la gran industria.

Este proceso de consolidación de la industria gráfica no tuvo una relación tan estrecha con el barrio. Desde sus inicios, el subsector estuvo ubicado principalmente en el centro de la ciudad, pero no específicamente en el sector de San Nicolás. Previamente, se localizaron en el barrio la editorial América (calle 15 \#2-14) y la editorial de Mariano Ramos (carrera 21 con calle 7), así como Carvajal S.A, en una zona periférica (carrera 5 con calle 15), pero no se podría considerar a San Nicolás, desde épocas tempranas, como un barrio de impresores. En la parte inicial del periodo que se está analizando, el barrio contaba con una diversidad muy amplia de subsectores económicos en su interior, tales como alimentos,

\footnotetext{
14 CVC, El Valle del Cauca en la economía nacional (Cali: Corporación Autónoma Regional del Valle del Cauca, 1975), 13. 15 DANE, Censo industrial 1944, 2036.

16 DANE, Censo industrial 1944.

17 DANE, Censo industrial 1971.
} 
bebidas, industria de la madera y fibras naturales, industria de la metalurgia y de los minerales no metálicos, industrias químicas, de los textiles, del vestido y de los servicios ${ }^{18}$.

\section{Barrio San Nicolás y el camino a la especialización espacial}

A comienzos de la década de 1940, el barrio San Nicolás era uno de los mejores reflejos del proceso de transición que estaba viviendo la ciudad de Cali; uno de los lugares de encuentro de dos culturas, de dos mundos, punto en el cual he insistido desde el inicio de este artículo. La convivencia entre tradición y modernidad, entre una vida citadina y campesina era latente en el barrio. Mientras proliferaban emisoras radiales, operaban diversas salas de cine y había una vehículos de tracción animal, y cómo pasaban rebaños de vacas por las calles del barrio ${ }^{19}$.

Al tiempo que el barrio contaba con una mayor actividad pública y que se abrían nuevos espacios de opinión y tertuliaderos, la vida religiosa continuaba siendo una práctica incuestionable dentro de la sociedad de ese momento. Así mismo, la mayor parte de la agitada vida política de la ciudad, la cual era liderada por diversos grupos políticos, se desarrollaba alrededor del parque principal del barrio: el 20 de Julio. Cabe anotar, no obstante, que todos estos rituales estaban atravesados por ambivalencias. Los habitantes del barrio, así como eran espectadores de las tertulias culturales y científicas que se desarrollaban en el edificio Bernal (calle 16 con carrera séptima) - un espacio donde se encontraban médicos, abogados, poetas y profesores del colegio Santa Librada-,

Figura 2.

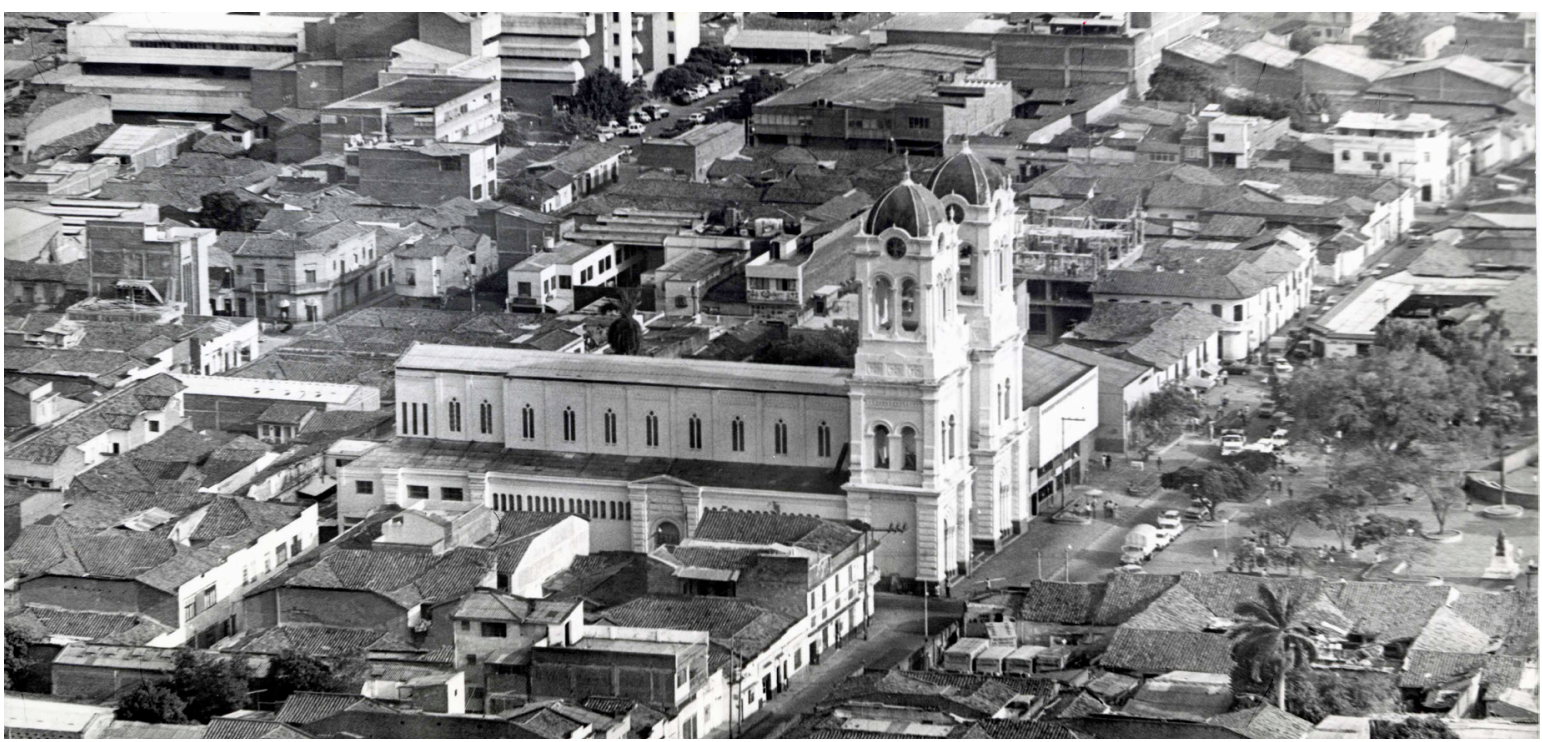

Fuente: Gustavo Gómez, «Vista aérea al barrio San Nicolás», Biblioteca Departamental Jorge Garcés Borrero, Archivo del Patrimonio Fotográfico y Fílmico de Valle del Cauca (Santiago de Cali, 1982).

intensa actividad pública, los habitantes del barrio veían cómo se mezclaba el tránsito de los automóviles modernos de la élite caleña con el de

18 En este punto es imprescindible agradecer al profesor Edgar Vásquez Benítez, quien me facilitó las listas de predios que elaboró para la década de 1940, basado en información de la Cámara de Comercio, de Historias de mi barrio y entrevistas. Más información sobre este tema, y en general sobre la historia del siglo XX en Santiago de Cali, se encuentra en su sugerente obra: Edgar Vásquez Benítez, Historia de Cali siglo XX: sociedad, economía, cultura y espacio (Cali: Artes Gráficas del Valle, 2001). igualmente escuchaban las míticas historias de uno de los personajes más famosos del barrio y de la ciudad, el famoso peluquero y sobador Alfonso Rivera, «Riverita», quien tenía su negocio frente al teatro Sucre ${ }^{20}$.

Las actividades en los espacios públicos del barrio $y$, especialmente, en el parque, fueron disminuyendo en la medida que las actividades en los

\footnotetext{
19 «Don Ari» (Seudónimo), San Nicolás (Cali: Concurso Recuerdos de mi barrio, 1984).

20 «on Ari», San Nicolás.
} 
espacios cerrados iban en aumento. Ante la práctica de sentarse en el parque, que fue perdiendo fuerza paulatinamente, se terminaron imponiendo el cine y los tertuliaderos en espacios cerrados, así como las reuniones para escuchar en la radio discursos políticos y radionovelas ${ }^{21} \mathrm{y}$, más avanzada la década de 1950, para ver televisión. Este cambio de prácticas culturales tuvo como telón de fondo uno de los momentos más nefastos de la historia de Colombia, el periodo conocido como la Violencia. Durante esta época, la ciudad no solo vio cómo se intensificó en el país el fenómeno de la violencia política, sino también el ascenso de los militares al poder y un proceso de acumulación de tierras desencadenado por la violencia rural, que a su vez contribuyó a uno de los procesos de urbanización más acelerados de la historia.

Otro aspecto que marcó la vida de la ciudad y transformó la del barrio sustancialmente fue la explosión del 7 de agosto de 1956, acontecimiento que golpeó directamente a San Nicolás. Como lo catalogaría uno de los testigos de primera fila, el padre Alfonso Hurtado Galvis, «fue una de las catástrofes más grandes por causas no naturales del hemisferio occidental» ${ }^{22}$. Lo cierto es que en Colombia no se había vivido un desastre de estas magnitudes. En horas de la madrugada, explotaron siete camiones cargados con dinamita, provenientes de Buenaventura, que se encontraban parqueados en la calle 25 , entre las carreras 1 y 4, dejando un saldo de 4.000 muertos y 12.000 heridos. Se estima que 41 manzanas quedaron

21 Esteban Morera Aparicio, «Vida pública antes y después del asesinato de Jorge Eliécer Gaitán», en Historia de Cali siglo XX, ed. Gilberto Loaiza (Cali: Universidad del Valle, 2012), 190-224.

22 Con relación a la explosión del 7 de agosto de 1956, hay una serie de trabajos audiovisuales, periodísticos y académicos que ilustran lo acontecido y que vale la pena consultar. Entre ellos se encuentran el documental de Juan Diego Mejía Una madrugada explosiva; los trabajos del profesor César Augusto Ayala: César Ayala Diago, «La explosión de Cali agosto 7 de 1956», Credencial Historia (1999), y «Mucho ruido y pocas nueces. A propósito de la explotación política de la explosión de Cali en agosto de 1956», Historia y Espacio, n. ${ }^{\circ} 16$ (2000): 7-20; y los especiales realizados por los periódicos de la ciudad. Cabe mencionar, sin embargo, que este es un acontecimiento que merece un tratamiento más profundo, y con el que la academia aún se encuentra en deuda. El hecho es abordado de manera tangencial en varios trabajos, pero también valdría la pena enfocarse en los diferentes fenómenos que se desprenden de él, no solo en relación con su impacto urbano, sino también con las consecuencias sociales y culturales que arrastró. destruidas, muchas de las cuales formaban parte de la zona de influencia del barrio San Nicolás. La plaza Belmonte, que jugaba uno de los papeles más importantes dentro de la economía del sector, a partir de este momento desapareció por completo de la historia de la ciudad. Lo mismo ocurrió con el teatro Roma, uno de los más concurridos por los sectores populares de San Nicolás, así como por los de los barrios adyacentes: Santander, Popular y Piloto. Con la reconstrucción, lo que era la plaza Belmonte pasó a convertirse en las modernas oficinas del Ingenio Riopaila y Colombina, una de las empresas de alimentos más grandes del país. El teatro Roma, por su parte, años después cedió sus terrenos a uno de los diarios más llamativos del siglo XX en Cali: El Caleño.

En esta fase de transición, el barrio San Nicolás presentó todos los fenómenos que estaba viviendo la ciudad, como resultado del proceso de industrialización que inició en la década de 1920 y que había transformado su vieja vocación artesanal. En la primera parte del periodo de estudio, el barrio contó con una presencia industrial fuerte, en la que aún no predominaban los impresores.

Adicionalmente, durante la primera mitad del siglo $\mathrm{XX}$ varios de los empresarios más importantes de la ciudad hicieron inversiones en el barrio. El libro del profesor Jairo Henry Arroyo, pionero en el estudio de las prácticas empresariales en Cali y el Valle del Cauca, muestra cómo Manuel María Buenaventura - quien lideró el proyecto del Teatro Municipal-, Hermann Sartore Bohmer - partícipe del proyecto del Teatro Municipal y líder del proyecto del Teatro Jorge Isaacs-, Julio Giraldo, Gabriel Garcés Borrero y Luis Fischer tenían varias propiedades en el barrio, entre las que se encontraban casas y lotes sin construirse ${ }^{23}$.

Los cambios que se presentaron en este periodo muestran cómo la industria va entrando en el barrio, en parte impulsada por el capital extranjero, que se evidenció con el establecimiento de las plantas de producción de las multinacionales del caucho, Goodyear (carrera 5a entre calles

\footnotetext{
23 Véase Jairo Henry Arroyo Reina, Historia de las prácticas empresariales en el Valle del Cauca: Cali 1900-1940 (Cali: Universidad del Valle, 2006), 166, 170, 268, 351.
} 
22 y 23) y Croydon (carrera 6a con calle 23$)^{24}$. Al mismo tiempo, la capacidad emprendedora de los sectores populares fue conformando una verdadera red productiva, que aprovechaba el entorno económico creado por las grandes y medianas industrias, y construyó una gran cantidad de tintorerías, panaderías, graneros, ventas de carne, droguerías, ebanisterías, pequeñas industrias de la confección y zapaterías ${ }^{25}$.

\section{La consolidación de la industria gráfica en el barrio San Nicolás}

Aunque el proceso de consolidación de San Nicolás como un barrio de impresores se comenzó a gestar entre las décadas de 1960 y 1970, varias industrias editoriales se habían asentado allí previamente, como es el caso de la editorial América - de la que se tienen registros desde la década de 1930-, de la editorial Belalcázar y de la editorial de Mariano Ramos — que se estableció desde finales de la década de 1950—. Antes de la década de 1960, la mayoría de las industrias de artes gráficas se encontraban ubicadas a lo largo del centro de la ciudad. Sin embargo, se hizo necesaria una especialización zonal dado el crecimiento urbano acelerado que, en el caso de Cali, fue impulsado mucho más por la dinámica económica del sector que por una política definida de planificación.

Las bases de datos de la Cámara de Comercio de Cali, así como las entrevistas realizadas, mostraron cómo a finales de los años sesenta y durante toda la década de 1970, la industria editorial comenzó a asentarse de manera más vigorosa en el barrio, en especial en las carreras 4 y 6 y en la calle $16^{26}$. Gran parte de estos establecimientos continuaron ocupando estos espacios en las décadas siguientes - posteriormente, los almacenes eléctricos y la fortaleza del centro comercial San Andresito van a desplazar a los impresores de algunos de sus espacios tradicionales-.

En este punto incidieron distintos aspectos que fueron definiendo la vocación económica del

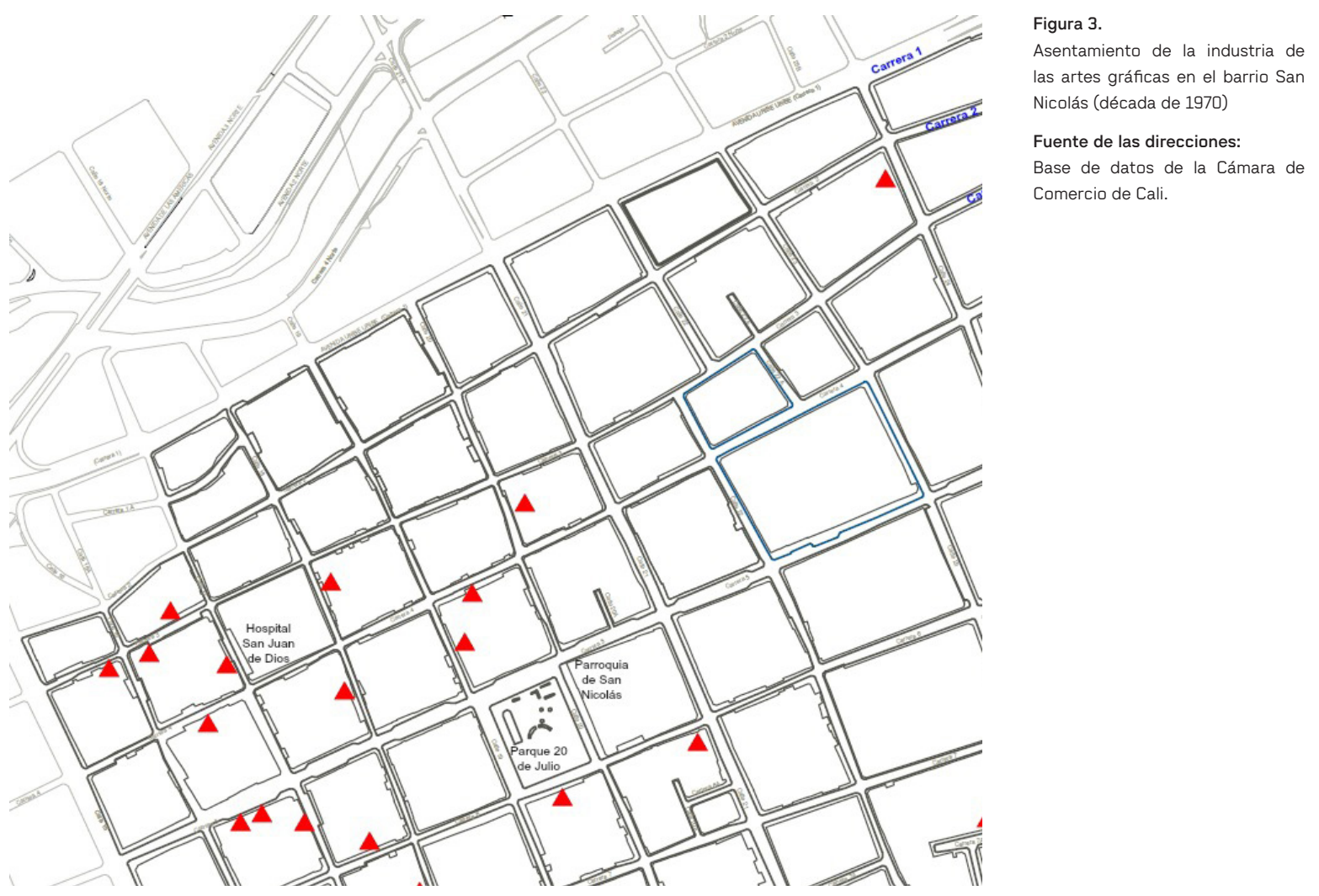

24 Vásquez Benítez, Historia de Cali, 186 y 196.

25 Grupo de Investigadores San Nicolás, San Nicolás (Cali: Concurso Recuerdos de mi barrio, 1984).

26 Javier Cardona, entrevistado por el Grupo seminario de Historia Cultural (Universidad Icesi), Cali, mayo de 2014.

El camino a la especialización espacial: el asentamiento de la industria de las artes gráficas / Esteban Morera Aparicio / 103 
barrio. Aunque un negocio tan diverso como el de las imprentas está marcado por una rica vida intelectual y política en su entorno, aquí solo me centraré en algunos aspectos propios de su dinámica económica, Para empezar, en la fortaleza de una de las empresas de industrias gráficas del país, Carvajal S.A., que desde su nacimiento se ubicó en una zona periférica del barrio, y que resultó clave para la consolidación de la industria editorial en la ciudad, ya que concentraba la mayor parte del negocio ${ }^{27}$. Esto cobra mayor importancia si se tiene en cuenta el lugar que ocupan las artes gráficas caleñas en la economía nacional y la articulación que se construyó con la también poderosa industria papelera vallecaucana.

Desde inicios del periodo de estudio, el papel de Carvajal S.A. dentro de la industria editorial de la región fue vital para la importación de insumos desde el exterior, circunstancia que aprovecharon las pequeñas y medianas industrias; téngase en cuenta que, dentro del proceso de producción editorial, las materias primas extranjeras eran sumamente importantes. Como lo podremos observar dentro de los censos industriales, en la década de 1940 alrededor del 95\% de las existencias de materias primas era de productos extranjeros ${ }^{28}$.

Para la década de 1970, Carvajal S.A. ya se había trasladado del centro hacia el norte de la ciudad —al lado de los talleres del ferrocarril en Chipichape- - Sin embargo, su influencia en el barrio no se detuvo en ese momento. Una gran parte de los trabajadores que habían formado parte de esta empresa, y que habían adquirido experiencia de la industria de las artes gráficas, comenzaron a conformar las pequeñas y medianas empresas que poblaron el barrio. Al mismo tiempo, en la capacidad de acceso a las tecnologías, Carvajal S.A. constituyó un puente entre la importación de máquinas y los pequeños productores, quienes constantemente le compraron a esta empresa maquinaria nueva y usada, para sus pequeños y medianos negocios ${ }^{29}$. Al igual que Carvajal S.A., la imprenta de Mariano Ramos, así como

27 Banco de la República, Informe económico del departamento del Valle del Cauca (Cali: Banco de la República de Colombia, 1974). 28 DANE, Censo industrial 1944, 2040-2041.

29 Cardona, entrevistado por el Grupo. las demás de la zona, eran auténticos espacios de formación de una profesión en la que el conocimiento empírico jugó un papel fundamental. Fueron constantes los casos de trabajadores que aprendieron empíricamente sobre el oficio en los diferentes talleres donde trabajaban, y que posteriormente, con estos conocimientos, lograron constituir sus propias empresas ${ }^{30}$.

Este fenómeno tomó mayor impulso en San Nicolás, gracias a la existencia de una estructura de economía popular que databa desde su formación como barrio artesano y que se fue adaptando paulatinamente a las nuevas estructuras económicas. Existía una red comercial e industrial de pequeños y medianos negocios que lograron articularse con la industria a gran escala que iba incursionando en el barrio. Gran parte de los trabajadores de las grandes industrias eran residentes del barrio, lo que facilitó el establecimiento de la red, y la mezcla del lugar de residencia con el de la actividad comercial. Aunque esta modalidad de establecimiento fue decayendo con el tiempo, en la década de 1980 aún había una cantidad significativa de negocios de artes gráficas que compartían la planta con el lugar de residencia ${ }^{31}$.

Otro aspecto que incidió en la agrupación de los diferentes talleres de artes gráficas en una zona específica de la ciudad, fue el nivel de subcontratación que exige este tipo de negocio. Por esta razón, se presentó un nivel de especialización fuerte dentro de cada uno de los talleres, que exigió la formación de diferentes redes productivas dentro del barrio, en las cuales pequeños, medianos y grandes negocios jugaron un papel dentro de la cadena ${ }^{32}$.

La gran cantidad de información que entregan los censos industriales, especialmente en sus primeras ediciones, nos permite ver cómo la maquinaria necesaria para completar los distintos pasos de los procesos de producción editorial estaba distribuida en diferentes locales. Solo las guillotinas y las perforadoras se encontraban en la mayoría de los

\footnotetext{
30 Omar Enrique Arango, entrevistado por el Grupo seminario de Historia Cultural (Universidad Icesi), junio de 2014.

31 Alcaldía de Santiago de Cali, Informe de caracterización subsectorial. Subsector de las artes gráficas (Cali: Alcaldía de Cali, 1997); Cardona, entrevistado por el Grupo; Elver Muñoz Ramos, entrevistado por el Grupo seminario de Historia Cultural (Universidad Icesi), mayo de 2014.

32 Alcaldía de Santiago de Cali, Informe de caracterización.
} 
establecimientos, en tanto que las 39 impresoras registradas en el Censo de 1944 estaban distribuidas en 13 de los 21 establecimientos de industrias gráficas. Los linotipos, por su parte, 15 en total, pertenecían a seis establecimientos. Así mismo, se pueden observar que existían establecimientos que concentraban una gran cantidad de maquinaria específica, como es el caso de las troqueladoras de etiquetas (120) y de sobres (37), que pertenecían a una sola empresa ${ }^{33}$. Todo esto muestra la dispersión de las actividades, la especialización de los establecimientos en los diferentes procesos y la eventual dependencia que tenían algunos establecimientos de otros que desarrollaban etapas distintas del proceso productivo, circunstancia que favoreció la agrupación de la industria gráfica en este sector de la ciudad. Esta situación no se alteró significativamente a lo largo del periodo, como lo muestran los censos industriales de las siguientes décadas, las entrevistas ${ }^{34}$ y los estudios que realizó la Alcaldía sobre el subsector de las artes gráficas ${ }^{35}$.

A pesar del crecimiento considerable que experimentó la industria de la maquinaria nacional dentro de este periodo (como lo podemos observar en la Tabla 3), una de las causas de esta división de las etapas de producción fue el alto costo de las maquinarias, el cual llevaba a que los pequeños empresarios solo pudieran adquirir una o dos, y, por ende, que tuvieran que dedicarse a una actividad específica. Así, ubicarse dentro de la red y estar en cercanía con los otros establecimientos jugó un papel vital en términos de tiempo y costos, para articular las diferentes fases de los procesos de impresión. Dentro de este marco se consolidó en el barrio una red dentro de los impresores, que facilitaba el acceso a la maquinaria y a los insumos, así como al trabajo. La capacidad asociativa de los impresores se materializó a mediados de la década de 1970 con el nacimiento de una cooperativa (Coimpresores) para facilitar el acceso a los insumos, proyecto que los impresores reseñaron como exitoso en la estabilización de los precios de las materias primas ${ }^{36}$.

33 DANE, Censo industrial 1944, 2054.

34 Cardona, entrevistado por el Grupo; Muñoz Ramos, entrevistado por el Grupo.

35 Alcaldía de Santiago de Cali, Informe de caracterización.

36 Arango, entrevistado por el Grupo.
A modo de síntesis se debe decir que el barrio San Nicolás fue convirtiéndose en el espacio más apropiado para el desempeño de las artes gráficas en la ciudad de Cali, ya que logró conjugar el desarrollo del mercado editorial y el aprovechamiento de las ventajas que ofrecieron las grandes empresas de artes gráficas, con la creatividad y percepción de los pequeños y medianos propietarios, quienes entendieron, gracias a su conocimiento de la industria y del potenciamiento de sus capacidades económicas y logísticas, en qué sector de la industria podrían lograr un mayor impacto comercial. Al mismo tiempo, ya sea por capacidad creativa o como un reflejo de sobrevivencia, estos empresarios lograron aprovechar al máximo sus capacidades económicas y convertirse en una industria que se consolidó con el paso del tiempo y que transformó gran parte de la vocación económica del barrio.

\section{Conclusiones}

Durante el periodo comprendido entre los años 1940 y 1980, observamos el asentamiento y la consolidación de la industria de las artes gráficas en el barrio San Nicolás. En un periodo de profundas transformaciones para la ciudad, dado que hubo un incremento de la actividad económica en todos los niveles, el barrio cumplió con un rol fundamental en la dinámica económica, así como en los procesos culturales de cambio.

Las causas de este fenómeno de especialización espacial no solo se encuentran en los cambios macroeconómicos que estaban sufriendo la ciudad y el país, sino que subyacen en el microcosmos que San Nicolás había desarrollado a lo largo de su historia como barrio artesanal, de obreros y microempresarios. La economía popular había tejido una compleja red a lo largo de los años que, con la aparición de las grandes empresas de artes gráficas, se convirtió en un escenario propicio para el asentamiento de pequeños, medianos y grandes impresores.

Todo esto nos plantea interrogantes en torno al papel que jugaron los barrios populares, no solo como espacios mediadores ante los cambios bruscos que estaban sufriendo las sociedades latinoamericanas en términos culturales, sino 
también cómo los barrios populares de tradición artesanal construyeron estructuras comerciales e industriales de pequeña escala, pero vigorosas, que se alimentaron con la llegada de las grandes empresas y que sirvieron para establecer puentes para el ingreso de los sectores populares en la economía capitalista. En el barrio San Nicolás, caso estudiado durante este artículo, dos factores fueron fundamentales, por un lado, los espacios que iban abriendo las grandes industrias - principalmente Carvajal S.A.-, al igual que la capacidad emprendedora de los sectores populares, quienes supieron potenciar sus capacidades para construir una red empresarial que se pudo consolidar a lo largo del tiempo.

\section{Bibliografía}

\section{Fuentes primarias}

Alcaldía de Santiago de Cali. Informe de caracterización subsectorial. Subsector de las artes gráficas. Cali: Alcaldía de Cali, 1997.

Arango, Omar Enrique. Entrevistado por el Grupo seminario de Historia Cultural (Universidad Icesi). Cali, junio de 2014.

Banco de la República. Informe económico del departamento del Valle del Cauca. Cali: Banco de la República de Colombia, 1974.

Cardona, Javier. Entrevistado por el Grupo seminario de Historia Cultural (Universidad Icesi). Cali, mayo de 2014.

CVC. El Valle del Cauca en la economía nacional. Cali: Corporación Autónoma Regional del Va1le del Cauca, 1975.

DANE. Censo Industrial 1944. Bogotá: Departamento Administrativo Nacional de Estadística, 1945.

DANE. Censo Industrial 1954. Bogotá: Departamento Administrativo Nacional de Estadística, 1955.
DANE. Censo Industrial 1971. Bogotá: Departamento Administrativo Nacional de Estadística, 1972.

«Don Ari» (Seudónimo). San Nicolás. Cali: Concurso Recuerdos de mi barrio, 1984.

Grupo de Investigadores San Nicolás. San Nicolás. Cali: Concurso Recuerdos de mi barrio, 1984.

Muñoz Ramos, Elver. Entrevistado por el Grupo seminario de Historia Cultural (Universidad Icesi). Cali, mayo de 2014.

\section{Fuentes secundarias}

Almario García, Oscar. «Cali y el Valle del Cauca: configuración moderna y reconfiguración contemporánea de la región y la ciudad región». En Historia de Cali siglo XX, tomo II, editado por Gilberto Loaiza, 70-93. Cali: Universidad del Valle, 2012.

Aprile-Gniset, Jacques. La ciudad colombiana: Siglo XIX y Siglo XX. Bogotá: Banco Popular, 1992.

Arroyo Reina, Jairo Henry. Historia de las prácticas empresariales en el Valle del Cauca: Cali 19001940. Cali: Universidad del Valle, 2006.

Ayala Diago, César. «La explosión de Cali, agosto 7 de 1956», Credencial Historia (1999).

Ayala Diago, César. «Mucho ruido y pocas nueces. A propósito de la explotación política de la explosión de Cali en agostos de 1956». Historia y Espacio, n. ${ }^{\circ} 16$ (2000): 7-20.

Bonilla, Ramiro. «Modelos urbanísticos de Cali en el siglo XX». En Historia de Cali, siglo XX, tomo I, editado por Gilberto Loaiza, 25-85. Santiago de Cali: Universidad del Valle, 2012.

Hurtado, Aura. «Opinión pública y formación del departamento del Valle, 1903-1910». CS 7 (2011): 161-192. 
Kalmanovitz, Salomón. «El desarrollo histórico del campo colombiano». En Colombia Hoy, editado por Jorge Orlando Melo González. Santa Fe de Bogotá: Presidencia de la República, 1996.

Mejía Pavony, Germán. Los años del cambio. Historia urbana de Bogotá. 1820-1910. Bogotá: Pontificia Universidad Javeriana, 2000.

Morera Aparicio, Esteban. «Vida pública antes y después del asesinato de Jorge Eliécer Gaitán». En Historia de Cali siglo XX, tomo II, editado por Gilberto Loaiza, 190-224. Cali: Universidad del Valle, 2012.

Morera Aparicio, Esteban. «Vida pública en Cali y el impacto del gaitanismo en la ciudad (1945 -1950)». Tesis de pregrado, Universidad del Va1le, 2011.

Planeación Municipal. Proceso histórico, periodos de formación de la ciudad. Cali: Alcaldía de Santiago de Cali, 1977.

Sáenz Rovner, Eduardo. La ofensiva empresarial: industriales, políticos y violencia en los años 40 en Colombia. Bogotá: Tercer Mundo, 1992.

Ulloa, Alejandro. «Modernidad, barrio popular y músicas mulatas: el samba brasilero y el tango ríoplatense». Nexus, Comunicación, n. ${ }^{\circ} 17$ (2015): 222-245.

Urrea Giraldo, Fernando. «Transformaciones sociodemográficas y grupos socioraciales en Cali, siglo XX e inicios del siglo XXI». En Historia de Cali siglo XX, tomo I, editado por Gilberto Loaiza Cano, 145-194. Cali: Universidad del Valle, 2012.

Vásquez Benítez, Édgar. Historia de Cali Siglo 20: sociedad, economia, cultura y espacio. Cali: Artes Gráficas del Valle, 2001.

Vásquez Benítez, Édgar. «Historia del desarrollo económico y urbano de Cali». Boletín socioeconómico Cidse, n. 20 (1990): 1-28.
Recibido: 18 de enero de 2016

Aceptado: 20 de marzo de 2016

Disponible en línea: 6 de diciembre de 2016

\section{Cómo citar este artículo}

Morera Aparicio, Esteban. «El camino a la especialización espacial: el asentamiento de la industria de las artes gráficas en el barrio San Nicolás. Santiago de Cali, 1940-1980». Memoria y Sociedad 20, n. 41 (2016): 93-107. http://dx.doi.org/10.11144/ Javeriana.mys20-41.ceea 